\title{
MAOAM: multiple-aspect outstandingness appraisement methodology
}

\section{Zghidi Imen}

\author{
MODILS Research Laboratory, \\ FSEG, \\ Sfax University, \\ Sfax, Tunisia \\ Email: zghidi.imen@gmail.com
}

\begin{abstract}
In this contribution, we devise and apply a method for ranking multi-attribute alternatives. The suggested method is founded, inter alia, on the so-called positive and negative outstandingness predicates. This method, herein called multiple-aspect outstandingness appraisement method (MAOAM), employs in the aggregation phase a family of multivariate generalised weighted Heronian means having as special cases the Beliakov et al.'s (2008) generalised Heronian means as well as that of Chu and Liu (2015). With positive and negative outstandingness predicates and multivariate generalised weighted Heronian means, we are better equipped to rank rationally pre-specified alternatives. The feasibility and accuracy of the proposed method are shown in a practical application.
\end{abstract}

Keywords: multi-criteria decision making; MCDM; positive outstandingness predicate; negative outstandingness predicate; weighted generalised Heronian mean operator.

Reference to this paper should be made as follows: Imen, Z. (2021) 'MAOAM: multiple-aspect outstandingness appraisement methodology', Int. J. Multicriteria Decision Making, Vol. 8, No. 3, pp.256-275.

Biographical notes: Zghidi Imen is currently a Lecturer at the ESEAC university, Sfax, Tunisia. She holds a Bachelor's in Operations Research and Production Planning in 2009, Master's in Operations Research in 2012, and a $\mathrm{PhD}$ in Quantitative Methods in 2016 from the University of Sfax, Tunisia. She is an author of some research articles on reasoning under uncertainty, statistical inference, and multi-criteria decision making.

\section{Introduction}

Multi-criteria decision making (MCDM) enables to select the most appropriate alternative among predetermined alternatives by evaluating them in terms of many criteria (Papathanasiou and Ploskas, 2018). According to recent literature (Kaya et al., 2019; Xing et al., 2018; Xu et al., 2018; Mao et al., 2018; Zhang et al., 2017; Zhu et al., 2019), there are different types of fuzzy MCDM methods and their applications in the literature such as: 
a pairwise comparison-based methods (e.g., analytic hierarchy process) where the decision makers can systematically assess the alternatives by making pair-wise comparisons for each of the chosen criteria (Saaty, 1980)

b outranking methods [e.g., PROMETHEE (Brans and Mareschal, 2005) and SIR $(\mathrm{Xu}, 2001)]$ in which - based on the deviation between the evaluations of every pair of alternatives in each criterion - aggregated preference indices and positive/negative or superiority/inferiority outranking flows are computed

c distance based methods [e.g., VIKOR and TOPSIS (Opricovic and Tzeng, 2007, 2004; San Cristóbal, 2011; Yazdani and Graeml, 2014)] in which alternatives are evaluated according to their distance to ideal solutions.

Nevertheless, expressing decision-makers' fuzzy decision information such as his preference information in an expressive and flexible manner remain a huge challenge. This is mainly due to the fact that subjective factors related to the decision-makers' own experience, his cognitive ability, and intuition hugely influence the decision information and hence the decision process and outcome.

Recently, tools and methods that can describe uncertain information and quite a few influential theories have been proposed. According to De Luca and Termini (1972), Weber (1984), Czogała et al. (1982), Farr and Brown (1971) and Ying (1990), there are three types of uncertainty when dealing with problems in fuzzy decision making:

1 randomness that can be captured by a probabilistic measure

2 fuzziness that can be measured using entropy or energy measures

3 alternativity, the opposite of outstandingness, that can be measured using the outstandingness measure introduced in Ying (1990).

Two types of outstandingness fuzzy binary predicates have been proposed in Ying (1990): a positive fuzzy predicate expressing the degree of positive outstandingness of one alternative over another whereas the negative fuzzy predicate expresses the degree of negative outstandingness of one alternative over another. A critical issue may, however, emerge from the process of subject judgments concerning appraisal behaviours when the MCDM approaches ignore one side of the evaluation. Indeed, the fuzzy set theory gives rise to type I bipolarity called the bipolar univariate model (Osgood, 1957). Fuzzy sets only give a membership degree to each element and the non-membership degree is always equivalent to the complementary. Therefore, a decrease in the negative degree (non-membership function) goes hand in hand with an increase of the positive degree (membership function). But, according to recent development in psychological studies, the positive and negative degrees are not necessarily complementary to each other (Cacioppo and Berntson, 1994; Cacioppo et al., 1997; Cacioppo and Berntson, 1999; Norris et al., 2010). An interesting property of the positive and negative outstandingness predicates is that they may be defined in such a way that they are antagonistic and completely independent of each other. Hence, such outstandingness predicates may exhibit a good ability to describe and express decision-makers' fuzzy decision information in an expressive and flexible manner that takes into consideration the latest development in psychological studies.

Another challenge in the design of MCDM methods is that attributes in most real problems are correlated. This led to a number of works in the literature to consider the 
application of the Bonferroni (1950) and Heronian means (Beliakov et al., 2008; Chu and Liu, 2015; Janous, 2001; $\mathrm{Yu}$ and $\mathrm{Wu}, 2012$ ) in the aggregation phase. According to a recent work Pramanik et al. (2020), however, the Heronian mean operator is shown to be better equipped to embody the interaction between attributes to avoid unreasonable situations in information aggregation. In Wang et al. (2017), the Bonferroni mean aggregation operator has been shown to neglect the relationship between each attribute and other attributes repeatedly when compared to the Hernonian mean. Furthermore, the Bonferroni aggregation operator has a larger computational complexity compared to the Heronian mean.

The main objective of this paper is to rank rationally pre-specified alternatives starting, from pairwise comparisons of alternative evaluations on each criterion that measure outstandingness. Towards this end, we make use of, among others, the fuzzy positive and negative outstandingness predicates which measure the degree of outsandingness of an alternative against another alternative in both the positive and negative senses (Ying, 1990). We give a number of various outstandingness predicates and study the properties of such predicate pairs. The proposed method, herein called multiple-aspect outstandingness appraisement method (MAOAM), employs in the aggregation phase, a family of multivariate generalised weighted Heronian means having as special cases the Beliakov et al.'s (2008) generalised Heronian means as well as that of Chu and Liu (2015). With positive and negative outstandingness predicates and multivariate generalised weighted Heronian means, we are better equipped to rank rationally pre-specified alternatives based on outstandingness that allow for

1 expressing the positive outstandingness degrees in an independent manner from the negative one

2 aggregating information that handles the possible interaction between attributes.

The rest of the paper is organised as follow. In Section 2, we define the problem and our assumptions. In Section 3, we introduce the concepts of positive and negative outstandingness predicates as well as the Heronian mean and its generalisations which constitute the mathematical tools for the MAOAM method. Next, the description of the MAOAM method as a ranking method well adapted to problems where a finite number of alternatives are to be ranked considering several criteria is presented in Section 4. Finally, before concluding in Section 6, we present an illustrative exampel using a real-world investment bank dataset in Section 5.

\section{Problem definition}

To begin, the problem formulation can be set out as follows: Given

- A set of $m$ feasible alternatives $A=\left\{A_{i} \mid i \in\{1, \ldots, m\}\right\}$.

- A set of $n$ aspects $C=\left\{C_{j} \mid j \in\{1, . ., n\}\right\}$.

- A $m \times n$ achievement (evaluation) matrix $a$ where $a_{i j}$ denotes the achievement value of alternative $A_{i}$ with respect to aspect $C_{j}$. Each line corresponds to an alternative and each column corresponds to an aspect. Each alternative has a vector of achievement (or degrees of satisfaction) $\in[0,1]^{n}$ unit hypercube. 


$$
\begin{aligned}
& \begin{array}{llll}
C_{1} & C_{2} & \ldots & C_{n}
\end{array} \\
& a=\begin{array}{c}
A_{1} \\
A_{2} \\
\vdots \\
A_{m}
\end{array}\left(\begin{array}{llll}
a_{1,1} & a_{1,2} & \ldots & a_{1, n} \\
a_{2,1} & a_{2,2} & \ldots & a_{2, n} \\
\vdots & \vdots & \vdots & \vdots \\
a_{m, 1} & a_{m, 2} & \ldots & a_{m, n}
\end{array}\right)
\end{aligned}
$$

Note that $\left(a_{i, 1}, a_{i, 2}, \ldots, a_{i, n}\right)$ is the achievement vector associated with alternative $A_{i}$ whereas $\left(a_{1, j}, a_{2, j}, \ldots, a_{m, j}\right)$ is the achievement vector associated with aspect $C_{j}$.

- $\quad$ A weight vector $w=\left(w_{1}, w 2, \ldots, w_{n}\right)$ which represents weights of relative importance of the different aspects. These weights are non-negative numbers independent from the measurement units of the aspect. The higher the weight, the more important is the aspect. Each aspect weight $w_{j}$ is in $(0,1)$ and

$$
1 \leq \sum_{j} w_{j} \leq n
$$

The objective is to rank the available alternatives using their positive and negative outstandingness predicates whose formulas will be set hereafter (Subsection 3.1)

\section{Development of MAOAM}

We introduce the concepts of positive and negative outstandingness predicates (Ying, 1990) as well as the Heronian mean and its generalisations which constitute the mathematical tools for the MAOAM method.

\subsection{Positive and negative outstandingness predicates}

Ying (1990) defined two fuzzy binary predicates on the unit interval $[0,1]$. One expresses the positive outstandingness of an alternative $x$ over alternative $y$, denoted by $\mathcal{P}(x, y)$, and the other for expressing the negative outstandingness, denoted by $\mathcal{N}(x, y)$.

Definition 3.1: A mapping $\mathcal{P}:[0,1] \times[0,1] \rightarrow[0,1]$ is a positive outstandingness predicate if and only if it satisfies the following properties.

$1 \mathcal{P}(1,0)=1$

2 if $x \leq y$, then $\mathcal{P}(x, y)=0$

$3 \mathrm{a} \quad$ if $y<x_{1}<x_{2}$, then $\mathcal{P}\left(x_{1}, y\right) \leq \mathcal{P}\left(x_{2}, y\right)$

$3 \mathrm{~b} \quad$ if $x_{2}<x_{1}<y$, then $\mathcal{P}\left(y, x_{1}\right) \leq \mathcal{P}\left(y, x_{2}\right)$.

The following predicates satisfy all four properties stated above and, hence, are all positive outstandingness predicates.

$$
\mathcal{P}_{1}(x, y)=\max (x-y, 0)
$$




$$
\begin{aligned}
& \mathcal{P}_{2}(x, y)= \begin{cases}1 & \text { if } x>y \\
0 & \text { otherwise. }\end{cases} \\
& \mathcal{P}_{3}(x, y)= \begin{cases}x & \text { if } x>y \\
0 & \text { otherwise. }\end{cases} \\
& \mathcal{P}_{4}(x, y)= \begin{cases}\frac{x-y}{x} & \text { if } x>y \\
0 & \text { otherwise. }\end{cases} \\
& \mathcal{P}_{5}(x, y)= \begin{cases}\frac{x}{x+y} & \text { if } x>y \\
0 & \text { otherwise. }\end{cases} \\
& \mathcal{P}_{6}(x, y)= \begin{cases}\frac{x-y}{x+y} & \text { if } x>y \\
0 & \text { otherwise. }\end{cases} \\
& \mathcal{P}_{7}(x, y)= \begin{cases}\frac{x-y}{1+\frac{y}{x}} & \text { if } x>y \\
0 & \text { otherwise. }\end{cases}
\end{aligned}
$$

It is important to mention that the generalised preference predicates defined as $P(d)$ where $d=x-y$ introduced in PROMETHEE (Brans and Mareschal, 2005) can also be viewed as positive outstandingness predicates for all constants $p, q, s \in[0,1]$ :

$$
\begin{aligned}
& \mathcal{P}_{2}(d)=\mathcal{P}_{2}(x, y)= \begin{cases}1 & \text { if } x>y \\
0 & \text { otherwise. }\end{cases} \\
& \mathcal{P}_{8}(x, y)= \begin{cases}1 & \text { if } x-y>q \\
0 & x-y \leq q\end{cases} \\
& \mathcal{P}_{9}(x, y)= \begin{cases}\frac{x-y}{p} & \text { if } x-y>p \\
0 & x-y-y \leq p\end{cases} \\
& \mathcal{P}_{10}(x, y)= \begin{cases}1 & \text { if } x-y>p \\
\frac{1}{2} & q \leq x-y \leq p \\
0 & x-y \leq q\end{cases} \\
& \mathcal{P}_{11}(x, y)= \begin{cases}1 & \text { if } x-y>p \\
\frac{x-y-q}{p-q} & q \leq x-y \leq p \\
0 & x-y \leq p\end{cases} \\
& \mathcal{P}_{12}(x, y)= \begin{cases}1-e^{-\frac{(x-y)^{2}}{2 s^{2}}} \quad \text { if } x-y>0 \\
0 & x-y \leq 0\end{cases}
\end{aligned}
$$

Definition 3.2: A mapping $\mathcal{N}:[0,1] \times[0,1] \rightarrow[0,1]$ is a negative outstandingness predicate if and only if it satisfies the following properties.

$1 \mathcal{N}(0,1)=1$

2 if $x \leq y$, then $\mathcal{N}(y, x)=0$ 
$3 \mathrm{a} \quad$ if $y<x_{1}<x_{2}$, then $\mathcal{N}\left(y, x_{1}\right) \leq \mathcal{N}\left(y, x_{2}\right)$

$3 \mathrm{~b} \quad$ if $x_{2}<x_{1}<y$, then $\mathcal{N}\left(x_{1}, y\right) \leq \mathcal{N}\left(x_{2}, y\right)$.

The following predicates satisfy all four properties stated above and, hence, are all negative outstandingness predicates.

$$
\begin{aligned}
& \mathcal{N}_{1}(y, x)=\max (x-y, 0) \\
& \mathcal{N}_{2}(y, x)= \begin{cases}0 & \text { if } y>x \\
1 & \text { otherwise. }\end{cases} \\
& \mathcal{N}_{3}(y, x)= \begin{cases}0 & \text { if } y>x \\
1-y & \text { otherwise. }\end{cases} \\
& \mathcal{N}_{4}(y, x)= \begin{cases}0 & \text { if } y \geq x \\
\frac{x-y}{x} & \text { otherwise. }\end{cases} \\
& \mathcal{N}_{5}(y, x)= \begin{cases}0 & \text { if } y \geq x \\
\frac{x}{x+y} & \text { otherwise. }\end{cases} \\
& \mathcal{N}_{6}(y, x)= \begin{cases}0 & \text { if } y \geq x \\
\frac{x-y}{x+y} & \text { otherwise. }\end{cases} \\
& \mathcal{N}_{7}(y, x)= \begin{cases}0 & \text { if } y \geq x \\
\frac{x-y}{1+\frac{y}{x}} & \text { otherwise. }\end{cases}
\end{aligned}
$$

Next, the following proposition states the compliment of a positive outstandingness predicate (Ying, 1990).

Proposition 1: If $\mathcal{P}$ is a positive outstandingness predicate, then $\mathcal{P}^{\mathrm{c}}$ defined by

$$
\mathcal{P}^{\mathrm{c}}(x, y)=\mathcal{P}(1-x, 1-y) \quad \forall x, y \in[0,1]
$$

is a negative outstandingness predicate.

Similarly, we can derive the compliment of a negative outstandingness predicate as follows (Ying, 1990):

Proposition 2: If $\mathcal{N}$ is a negative outstandingness predicate, then $\mathcal{N}^{c}$ defined by

$$
\mathcal{N}^{c}(x, y)=\mathcal{N}(1-x, 1-y) \quad \forall x, y \in[0,1]
$$

is a positive outstandingness predicate.

Definition 3.3: Let $\mathcal{P}$ be a positive outstandingness predicate and $\mathcal{N}$ is a negative outstandingness predicate such that:

$$
\mathcal{N}(x, y)=\mathcal{P}(1-x, 1-y) \quad \forall x, y \in[0,1]
$$

and

$$
\mathcal{P}(x, y)=\mathcal{N}(1-x, 1-y) \quad \forall x, y \in[0,1]
$$


Then, $(\mathcal{P}, \mathcal{N})$ are said to be double pair predicates denoted D-pair.

In addition to the D-pair, we introduce four more pairs of the outstandingness predicates.

Definition 3.4: If $\mathcal{P}(x, y)=\mathcal{N}(y, x) \forall x, y \in[0,1]$ then $(\mathcal{P}, \mathcal{N})$ are called unbiased pair predicates denoted by U-pair.

For instance, $\left(\mathcal{P}_{1}, \mathcal{N}_{1}\right)$ is a U-pair. Consider the generalised preference predicates $\mathcal{P}_{2}$, $\mathcal{P}_{8}, \mathcal{P}_{9}, \mathcal{P}_{10}, \mathcal{P}_{11}$, and $\mathcal{P}_{12}$ introduced in PROMETHEE (Brans and Mareschal, 2005) to compute the preference of alternative $x$ over alternative $y$. To compute the preference of alternative $y$ over $x$ instead (from which negative outranking flow are computed), the PROMETHEE method simply swaps the inputs of $\mathcal{P}(x, y)$, i.e., it uses $\mathcal{P}(y, x)$. Since $\mathcal{P}(x, y)$ is a positive outstandingness predicate, it can easily be shown that $\mathcal{P}(y, x)$ is indeed a negative outstandingness predicate. Thus, in the PROMETHEE method it turns out that only $U$-pairs of the form $(\mathcal{P}(x, y), \mathcal{P}(y, x))$ are indeed used to compute the poditive/negative preference index between pairs of alternatives in each criterion.

It is important to note that U-pairs are not independent of each other and do provide complimentary evaluations. Next, we introduce pairs whose positive and negative evaluations are independent.

Definition 3.5: If $\mathcal{P}(x, y) \leq \mathcal{N}(y, x) \forall x, y \in[0,1]$ then $(\mathcal{P}, \mathcal{N})$ are called negativity biais pair predicates denoted by NB-pair.

For instance, $\left(\mathcal{P}_{5}, \mathcal{N}_{2}\right)$ is a NB-pair.

Definition 3.6: If $\mathcal{P}(x, y) \geq \mathcal{N}(y, x) \forall x, y \in[0,1]$ then $(\mathcal{P}, \mathcal{N})$ are called positivity biais pair predicates denoted by $\mathrm{PB}-$ pair.

For instance, $\left(\mathcal{P}_{4}, \mathcal{N}_{1}\right)$ is a PB-pair.

Definition 3.7: If all the previous conditions are not satisfied, then $(\mathcal{P}, \mathcal{N})$ are called arbitrary pair predicates denoted by A-pair.

For instance, $\left(\mathcal{P}_{3}, \mathcal{N}_{3}\right)$ is an A-pair.

\subsection{The Heronian mean and its generalisations}

Let $a$ and $b$ be two non-negative real numbers. The Heronian mean of $a$ and $b$ is defined in equation (1) as follows (Beliakov et al., 2008):

$$
H e(a, b)=\frac{a+\sqrt{a b}+b}{3}
$$

Recently, Janous (2001) defined further the generalised Heronian mean as shown in equation (2):

$$
H_{\omega}(a, b)= \begin{cases}\frac{a+\omega \sqrt{a b}+b}{\omega+2} & 0 \leq \omega \leq+\infty \\ \sqrt{a b} & \omega=+\infty\end{cases}
$$


Note that if $\omega=1$, it is clear that $H_{1}(a, b)=H e(a, b)$. If $\omega=0$, then $H_{0}(a, b)=\frac{a+b}{2}=$ $A(a, b)$ where $A(a, b)$ is the arithmetic mean. If $\omega=\infty$, then $H_{\infty}(a, b)=\sqrt{a b}=$ $G(a, b)$ where $G(a, b)$ is the geometric mean.

$$
G(a, b) \leq H_{\omega}(a, b) \leq A(a, b)
$$

Note that the above Heronian mean and its generalisations are those of two real numbers. Next, we will define the generalised Heronian mean in $n$ variables:

Let $x=\left(x_{1}, x_{2}, \ldots, x_{n}\right) ; x_{i} \geq 0, i=1,2, \ldots, n$. The generalised Heronian mean in $n$ variables $x_{1}, x_{2}, \ldots, x_{n}$ is defined in equation (4).

$$
H_{\omega}(x) \begin{cases}\sum_{i} x_{i}+\omega \sqrt[n]{x_{1} x_{2} \ldots x_{n}} & 0 \leq \omega<+\infty \\ \sqrt[n]{x_{1} x_{2} \ldots x_{n}} & \omega=+\infty\end{cases}
$$

Note that if $n=2, H_{\omega}(x)=H_{\omega}(a, b)$.

Beliakov et al. (2008) has proposed a different generalisation of the Hernian mean in $x$ which does not allow for $\sqrt[n]{x_{1} x_{2} \ldots x_{n}}$ to evaluate to zero if any of the $x_{i}$ is zero and is shown in equation (5).

$$
H R(x)=\frac{2}{n(n+1)} \sum_{i=1}^{n} \sum_{j=1}^{n} \sqrt{x_{i} x_{j}}
$$

Note that for $n=2$, we have $H R(a, b)=H e(a, b)$.

We might as well extend Beliakov's definition to introduce $\omega$ as in Janous (2001) and the extended form is shown in equation (6):

$$
H R(x, \omega)=\frac{\sum_{k=1}^{n} x_{k}+\omega \sum_{k=1}^{n} \sum_{l=k+1}^{n} \sqrt{x_{i} x_{j}}}{n+\omega \frac{n(n-1)}{2}}
$$

Note that $\operatorname{HR}(x, 1)=H R(x)$.

Unfortunately, the Heronian mean $H R(x)$ and the generalised Heronian mean $H_{\omega}(x)$ operators ignore the weights of the criteria which has been incorporated in the generalisation of $\mathrm{Chu}$ and Liu (2015) called the weighted generalised Heronian mean $(W G H M)$. Let $W=\left(w_{1}, w_{2}, \ldots, w_{n}\right)$ be the relative importance weights of $x=\left(x_{1}, x_{2}, \ldots, x_{n}\right)$ such that $\sum_{i=1}^{n} w_{i}=1$, respectively. Let $p, q \geq 0$. The $W G H M^{p, q}(x, W)$ defined in Chu and Liu (2015) in shown in equation (7):

$$
W G H M^{p, q}(x, W)=\frac{\left(\sum_{k=1}^{n} \sum_{l=1}^{n}\left(w_{k} x_{k}\right)^{p}\left(w_{l} x_{l}\right)^{q}\right)^{\frac{1}{p+q}}}{\left(\sum_{k=1}^{n} \sum_{l=k}^{n} w_{k}^{p} w_{l}^{q}\right)^{\frac{1}{p+q}}}
$$

In the following, we extend the $W G H M$ operator by taking into account $\omega$ (as in Walher Janous) as well as weights.

Let $W=\left(w_{1}, w_{2}, \ldots, w_{n}\right)$ the relative importance weights of $x=\left(x_{1}, x_{2}, \ldots, x_{n}\right)$, respectively. Let $p, q \geq 0$. Let $|W|=\sum_{k=1}^{n} w_{k}$ be the magnitude of $W$. We propose a generalisation of the Hernian mean, named $W G H M$, in $n$ variables that takes into consideration the relative importance weights as follows. 
When $0 \leq \omega<+\infty$, we have equation (8).

$$
\begin{aligned}
& W \operatorname{WH}_{\omega}^{p, q}(x, W) \\
& =\frac{\left(\sum_{k=1}^{n}\left(w_{k} x_{k}\right)^{p+q}+\omega \sum_{k=1}^{n} \sum_{l=k+1}^{n}\left(w_{k} x_{k}\right)^{p}\left(w_{l} x_{l}\right)^{q}\right)^{\frac{1}{p+q}}}{\left(\sum_{k=1}^{n} w_{k}^{p+q}+\omega \sum_{k=1}^{n} \sum_{l=k+1}^{n} w_{k}^{p} w_{l}^{q}\right)^{\frac{1}{p+q}}}
\end{aligned}
$$

When $\omega=+\infty$, we have equation (9).

$$
W \operatorname{WH}_{\omega}^{p, q}(x, W)=\frac{\left(\sum_{k=1}^{n} \sum_{l=k+1}^{n}\left(w_{k} x_{k}\right)^{p}\left(w_{l} x_{l}\right)^{q}\right)^{\frac{1}{p+q}}}{\left(\sum_{k=1}^{n} \sum_{l=k+1}^{n} w_{k}^{p} w_{l}^{q}\right)^{\frac{1}{p+q}}}
$$

If the sum of the weights is one and $\omega=1$, we have:

$$
W G H M_{\omega}^{p, q}(x, W)=W G H M^{p, q}(x, W)
$$

If the relative importance weights are equal and $p=q=\frac{1}{2}$, we have:

$$
\begin{aligned}
& W G H M_{\omega}^{\frac{1}{2}, \frac{1}{2}}(x, W)=H R(x, \omega) \\
& W G H M_{\omega=1}^{\frac{1}{2}, \frac{1}{2}}(x, W)=H R(x) \\
& W G H M_{\omega}^{\frac{1}{2}, \frac{1}{2}}\left(\left(x_{1}, x_{2}\right), W\right)=H_{\omega}\left(x_{1}, x_{2}\right)
\end{aligned}
$$

In the following, we prove that $W G H M_{\omega}^{p, q}(x, W)$ has the properties of idempotency, monotonicity, and internality.

Theorem 3: $W G H M_{\omega}^{p, q}(x, W)$ is idempotent.

Proof: Let $x_{i}=a$ for all $i=1,2, \ldots, n$.

$$
\begin{aligned}
W G H M_{\omega}^{p, q}(x, W) & =\frac{\left(\sum_{k=1}^{n} w_{k}^{p+q} a^{p+q}+\omega \sum_{k=1}^{n} \sum_{l=k+1}^{n} w_{k}^{p} w_{l}^{q} a^{p+q}\right)^{\frac{1}{p+q}}}{\left(\sum_{k=1}^{n} w_{k}^{p+q}+\omega \sum_{k=1}^{n} \sum_{l=k+1}^{n} w_{k}^{p} w_{l}^{q}\right)^{\frac{1}{p+q}}} \\
& =\frac{\left(a^{p+q}\left(\sum_{k=1}^{n} w_{k}^{p+q}+\omega \sum_{k=1}^{n} \sum_{l=k+1}^{n} w_{k}^{p} w_{l}^{q}\right)\right)^{\frac{1}{p+q}}}{\left(\sum_{k=1}^{n} w_{i}^{p+q}+\omega \sum_{k=1}^{n} \sum_{l=k+1}^{n} w_{k}^{p} w_{l}^{q}\right)^{\frac{1}{p+q}}} \\
& =a \frac{\left(\sum_{k=1}^{n} w_{k}^{p+q}+\omega \sum_{k=1}^{n} \sum_{l=k+1}^{n} w_{k}^{p} w_{l}^{q}\right)^{\frac{1}{p+q}}}{\left(\sum_{k=1}^{n} w_{k}^{p+q}+\omega \sum_{k=1}^{n} \sum_{l=k+1}^{n} w_{k}^{p} w_{l}^{q}\right)^{\frac{1}{p+q}}} \\
& =a \frac{\left(\sum_{k=1}^{n} w_{k}^{p+q}+\omega \sum_{k=1}^{n} \sum_{l=k+1}^{n} w_{k}^{p} w_{l}^{q}\right)^{\frac{1}{p+q}}}{\left(\sum_{k=1}^{n} w_{k}^{p+q}+\omega \sum_{k=1}^{n} \sum_{l=k+1}^{n} w_{k}^{p} w_{l}^{q}\right)^{\frac{1}{p+q}}} \\
& =a
\end{aligned}
$$

Theorem 4: $W G H M_{\omega}^{p, q}(x, W)$ is monotone. 
Proof: Let $x=\left(x_{1}, x_{2}, \ldots, x_{n}\right)$ and $y=\left(y_{1}, y_{2}, \ldots, y_{n}\right)$ such that $x_{k} \geq y_{k}$ for all $k$. We need to show that $W G H M_{\omega}^{p, q}(x, W) \geq W G H M_{\omega}^{p, q}(y, W)$.

Consider

$$
W \operatorname{WH}_{\omega}^{p, q}(x, W)=\frac{\left(\sum_{k=1}^{n}\left(w_{k} x_{k}\right)^{p+q}+\omega \sum_{k=1}^{n} \sum_{l=k+1}^{n}\left(w_{k} x_{k}\right)^{p}\left(w_{l} x_{l}\right)^{q}\right)^{\frac{1}{p+q}}}{\left(\sum_{k=1}^{n} w_{k}^{p+q}+\omega \sum_{k=1}^{n} \sum_{l=k+1}^{n} w_{k}^{p} w_{l}^{q}\right)^{\frac{1}{p+q}}}
$$

Since, $p, q \geq 0,0 \leq \omega \leq+\infty$, and $x_{k} \geq y_{k}$, we have $\left(w_{k} x_{k}\right)^{p+q} \geq\left(w_{k} y_{k}\right)^{p+q}$. Thus,

$$
W \operatorname{WH}_{\omega}^{p, q}(x, W) \geq \frac{\left(\sum_{k=1}^{n}\left(w_{k} y_{k}\right)^{p+q}+\omega \sum_{k=1}^{n} \sum_{l=k+1}^{n}\left(w_{k} x_{k}\right)^{p}\left(w_{l} x_{l}\right)^{q}\right)^{\frac{1}{p+q}}}{\left(\sum_{k=1}^{n} w_{k}^{p+q}+\omega \sum_{k=1}^{n} \sum_{l=k+1}^{n} w_{k}^{p} w_{l}^{q}\right)^{\frac{1}{p+q}}}
$$

Similarly, because $\left(w_{l} x_{l}\right)^{p} \geq\left(w_{k} y_{k}\right)^{p}$ and $\left(w_{l} x_{l}\right)^{q} \geq\left(w_{l} y_{l}\right)^{q}$ we have

$$
W \operatorname{WH}_{\omega}^{p, q}(x, W) \geq \frac{\left(\sum_{k=1}^{n}\left(w_{k} y_{k}\right)^{p+q}+\omega \sum_{k=1}^{n} \sum_{l=k+1}^{n}\left(w_{k} y_{k}\right)^{p}\left(w_{l} y_{l}\right)^{q}\right)^{\frac{1}{p+q}}}{\left(\sum_{k=1}^{n} w_{k}^{p+q}+\omega \sum_{k=1}^{n} \sum_{l=k+1}^{n} w_{k}^{p} w_{l}^{q}\right)^{\frac{1}{p+q}}}
$$

Thus, $W G H M_{\omega}^{p, q}(x, W) \geq W G H M_{\omega}^{p, q}(y, W)$.

Theorem 5: $W G H M_{\omega}^{p, q}(x, W)$ satisfies the internality property.

Proof: Let $x^{-}=\min \left(x_{1}, x_{2}, \ldots, x_{n}\right)$ and $x^{+}=\max \left(x_{1}, x_{2}, \ldots, x_{n}\right)$. According to Theorem 1, we have

$$
\begin{aligned}
& W \operatorname{WHM}_{\omega}^{p, q}\left(\left(x^{-}, x^{-}, \ldots, x^{-}\right), W\right)=x^{-} \\
& W G H M_{\omega}^{p, q}\left(\left(x^{+}, x^{+}, \ldots, x^{+}\right), W\right)=x^{+}
\end{aligned}
$$

Since, $x^{-} \leq x_{k} \leq x^{+}$for all $k$, we have

$$
\begin{aligned}
& W G H M_{\omega}^{p, q}\left(\left(x^{-}, x^{-}, \ldots, x^{-}\right), W\right) \\
& \leq W G H M_{\omega}^{p, q}(x, W) \\
& \leq W G H M_{\omega}^{p, q}\left(\left(x^{+}, x^{+}, \ldots, x^{+}\right), W\right)
\end{aligned}
$$

Thus,

$$
x^{-} \leq W G H M_{\omega}^{p, q}(x, W) \leq x^{+}
$$

In this section, we have introduced two generalisation of the Heronian mean. The first one denoted $H R(x, \omega)$ extends Beliakov's definition by introducing the parameter $\omega$ as in Janous (2001) and the extended form is shown in equation (6). The introduction of $\omega$ provides more flexibility to the decision-maker since when $\omega$ is set to zero, $H R(x, \omega)$ becomes the arithmetic mean, and as $\omega$ gets larger and larger $(\omega \rightsquigarrow \infty)$, it approaches the geometric mean. For the other values of $\omega$, we have a wide range of Heronian means. The second generalisation extends the $W G H M$ operator by taking into account $\omega$ (as in Walher Janous) as well as weights [denoted by $W G H M_{\omega}^{p, q}(x, W)$ and shown in equations (8) and (9)]. The advantage of this generalisation, like $H R(x, \omega)$, is the introduction of the paramter $\omega$ which allows it express various means (arithmetic, geometric, and Heronian). Furthermore, it also takes into consideration the relative weights when aggregating the information. These features allow it to be an expressive and flexible aggregation operator. 


\section{The MAOAM method}

MAOAM is a ranking method well adapted to problems where a finite number of alternatives are to be ranked considering several criteria. Recall that we are given,

- $\quad$ A set of $m$ feasible alternatives $A=\left\{A_{i} \mid i \in\{1, \ldots, m\}\right\}$.

- A set of $n$ aspects $C=\left\{C_{j} \mid j \in\{1, \ldots, n\}\right\}$.

- A $m \times n$ achievement (evaluation) matrix $a$ where $a_{i j}$ denotes the achievement value of alternative $A_{i}$ with respect to aspect $C_{j}$. Each line corresponds to an alternative and each column corresponds to an aspect. Each alternative has a vector of achievement (or degrees of satisfaction) $\in[0,1]^{n}$ unit hypercube.

- $\quad$ A weight vector $w=\left(w_{1}, w 2, \ldots, w_{n}\right)$ which represents weights of relative importance of the different aspects. These weights are non-negative numbers independent from the measurement units of the aspect. The higher the weight, the more important is the aspect. Each aspect weight $w_{j}$ is in $(0,1)$ and

$$
1 \leq \sum_{j} w_{j} \leq n
$$

The objective is to rank the available alternatives using their positive and negative outstandingness predicates. The procedural steps of the parameterised MAOAM method are enlisted below:

In our first step, we make pairwise comparisons between all the alternatives for each aspect using positive outstandigness predicates (POP) and negative outstandigness predicates (NOP). Assume we have a POP $\mathcal{P}($ resp. NOP $\mathcal{N})$. For each aspect $C_{j}$ and for each alternative $A_{k}$, we compute the positive (resp. negative) outstandingness of $A_{k}$ against every other alternative $A_{l}\left(\neq A_{k}\right)$ denoted by $P O_{k, l}^{j}$ (resp. $\left.N O_{k, l}^{j}\right)$ as follows:

$$
\begin{aligned}
& P O_{k, l}^{j}=\mathcal{P}\left(a_{k, j}, a_{l, j}\right) \forall j \in\{1, \ldots, n\}, \forall k \in\{1, \ldots, m\}, \forall l \in\{1, \ldots, m \mid l \neq k\} \\
& N O_{k, l}^{j}=\mathcal{N}\left(a_{k, j}, a_{l, j}\right) \forall j \in\{1, \ldots, n\}, \forall k \in\{1, \ldots, m\}, \forall l \in\{1, \ldots, m \mid l \neq k\}
\end{aligned}
$$

For each aspect $j$ and alternative $k$, let

$$
P O_{k}^{j}=\left(P O_{k, l}^{1}, \ldots, P O_{k, m}^{j}\right)
$$

where $\forall l \in\{1, \ldots, m \mid l \neq k\}$.

Similarity, let

$$
N O_{k}^{j}=\left(N O_{k, l}^{1}, \ldots, N O_{k, m}^{j}\right)
$$

Our second step is to compute the partial positive outstandingness ${ }^{+} \Omega_{k}^{j}$ using the extended Hernonian mean of Beliakov et al. (2008) introduced in Section 3:

$$
{ }^{+} \Omega_{k}^{j}=H R\left(P O_{k}^{j}, \omega\right)
$$

Similarly, we compute the partial negative outstandingness $-\Omega_{k}^{j}$ as follows:

$$
{ }_{-} \Omega_{k}^{j}=H R\left(N O_{k}^{j}, \omega\right)
$$


In the third step, we compute ${ }^{+} \Omega_{k}$ as the global positive outstandingness index of each alternative $k$ by using our generalised hernonian mean as follows:

$$
{ }^{+} \Omega_{k}=W G H M_{\omega}^{p, q}\left(\left({ }^{+} \Omega_{k}^{1}, \ldots,{ }^{+} \Omega_{k}^{j}, \ldots,{ }^{+} \Omega_{k}^{n}\right), W\right)
$$

Similarly, we compute $-\Omega_{k}$ as the global negative outstandingness index of each alternative $k$ as follows:

$$
{ }^{-} \Omega_{k}=W G H M_{\omega}^{p, q}\left(\left(-\Omega_{k}^{1}, \ldots,{ }^{-} \Omega_{k}^{j}, \ldots,{ }^{-} \Omega_{k}^{n}\right), W\right)
$$

In the fourth step, we calculate the bounded difference overall outstandigness for each alternative $k$ as follows:

$$
\Omega_{k}=\max \left(0,1-\frac{-\Omega_{k}}{+\Omega_{k}}\right)
$$

Or, alternatively, we compute the net outstandingness for each alternative $k$ as follows:

$$
\Omega_{k}={ }^{+} \Omega_{k}-{ }^{-} \Omega_{k}
$$

Finally, in step five, we determine the ranking of all alternatives depending on the values of $\Omega_{k}$. The higher value of $\Omega_{k}$, the more outstanding is the alternative.

\section{Illustrative example}

In this section, we validate the properties and evaluate the sensitivity of the MAOAM method using a real-life use case by mainly varying the:

- Outstandingness predicates: We consider four pairs of oustandingness predicates: an unbiased U-pair $((P 1, N 1))$, a negatively biased NB-pair $((P 5, N 2))$, a positively biased U-pair $((P 4, N 1))$, and an arbitrary A-pair $((P 3, N 3))$. This variation will allow us to validate the properties of these different outstandingness predicates.

- Heronian means parameters: In the MAOAM method, we introduced two Heronian means in the second and third step where each operator is parameterised with $\omega$. Here, we use $\omega_{1}$ instead of $\omega$ in the aggregation operator in the second step and $\omega_{2}$ instead of $\omega$ in the third step to remove any ambiguity since each aggregation in each step can use different values for $\omega$. We then consider values $\omega_{1}, \omega_{2} \in\{0,1,000\}$ that allows us to consider extreme cases when the aggregation is compensatory (when $\omega_{1}=\omega_{2}=0$ ) and when the aggregation is non-compensatory (when $\omega_{1}=\omega_{2}=1,000$ ) and combinations of these two extreme cases. This variation will allow us to study the sensitivity of the MAOAM method to different instantiations of the proposed Heronian means.

\subsection{Context}

The managers of a Tunsian investment bank receive applications of ivestment loans from various businessmen. The criteria alongside their weights used to evaluate the alternatives are shown in Table 1.

There are 32 candidates and their respective evaluations on the 18 criteria are shown in Table 3 where the objective is to rank the candidates according to their 'credibility' on a set of 18 weighted criteria. 
Table 1 The criteria and their corresponding weights

\begin{tabular}{lcc}
\hline & Criterion & Weight \\
\hline$C_{1}$ & Quality of the project proposal & 0.075 \\
$C_{2}$ & Applicant's responsiveness & 0.05 \\
$C_{3}$ & Applicant's risk aversion & 0.075 \\
$C_{4}$ & Administrative and financial management & 0.12 \\
$C_{5}$ & Technical knowledge & 0.12 \\
$C_{6}$ & Commercial position & 0.06 \\
$C_{7}$ & Infrastructure and logistics risk & 0.025 \\
$C_{8}$ & Technology risk & 0.05 \\
$C_{9}$ & Supply risk & 0.05 \\
$C_{10}$ & Regulation risk & 0.025 \\
$C_{11}$ & Demand risk & 0.05 \\
$C_{12}$ & Offer risk & 0.025 \\
$C_{13}$ & Quality risk & 0.05 \\
$C_{14}$ & Distribution risk & 0.025 \\
$C_{15}$ & Financial risk & 0.05 \\
$C_{16}$ & Customer risk & 0.05 \\
$C_{17}$ & Profitability risk & 0.05 \\
$C_{18}$ & Financial exploitation risk & 0.05 \\
\hline
\end{tabular}

Table 2 Values of the measure A

\begin{tabular}{lcccc}
\hline & $U$-pair $(P 1, N 1)$ & NB-pair $(P 5, N 2)$ & PB-pair (P4, N1) A-pair (P3, N3) \\
\hline$\omega_{1}=\omega_{2}=0$ & 16 & 2 & 20 & 16 \\
$\omega_{1}=0, \omega_{2}=1,000$ & 16 & 2 & 20 & 16 \\
$\omega_{1}=1,000, \omega_{2}=0$ & 16 & 2 & 17 & 16 \\
$\omega_{1}=1,000, \omega_{2}=1,000$ & 16 & 2 & 16 & 16 \\
\hline
\end{tabular}

\subsection{Results}

The results of are shown in Table $4\left(\omega_{1}=\omega_{2}=0\right)$, Table $5\left(\omega_{1}=0, \omega_{2}=1,000\right)$, Table $6\left(\omega_{1}=1,000, \omega_{2}=0\right)$, and Table $7\left(\omega_{1}=\omega_{2}=1,000\right)$ where we report, for each alternative $k$ and for each oustandingness predicate pair, the net oustandingess value $\Omega_{k}$ and the alternative's rank. Note that, we set $p=q=\frac{1}{2}$ for all experiments.

To validate the properties of the various fuzzy outstandingness predicate pairs, we introduce the following measure, denoted measure $A$ : which is the number of alternatives with positive net outstandingness divided by the total number of alternatives. We summarise the results of measure $A$ in Table 2 which confirm that the positively biased pair weighs most the positive outstandingness of an alternative wrt the negative one, followed by the U-pair and the A-pair and the negatively biased pair weighs most the negative outstandingness of an alternative instead. Furthermore, on this example, the only measure $A$ that is not stable across different configurations of the $\omega$ 's is the PB-pair $(P 4, N 1)$. 
Table 3 Input data

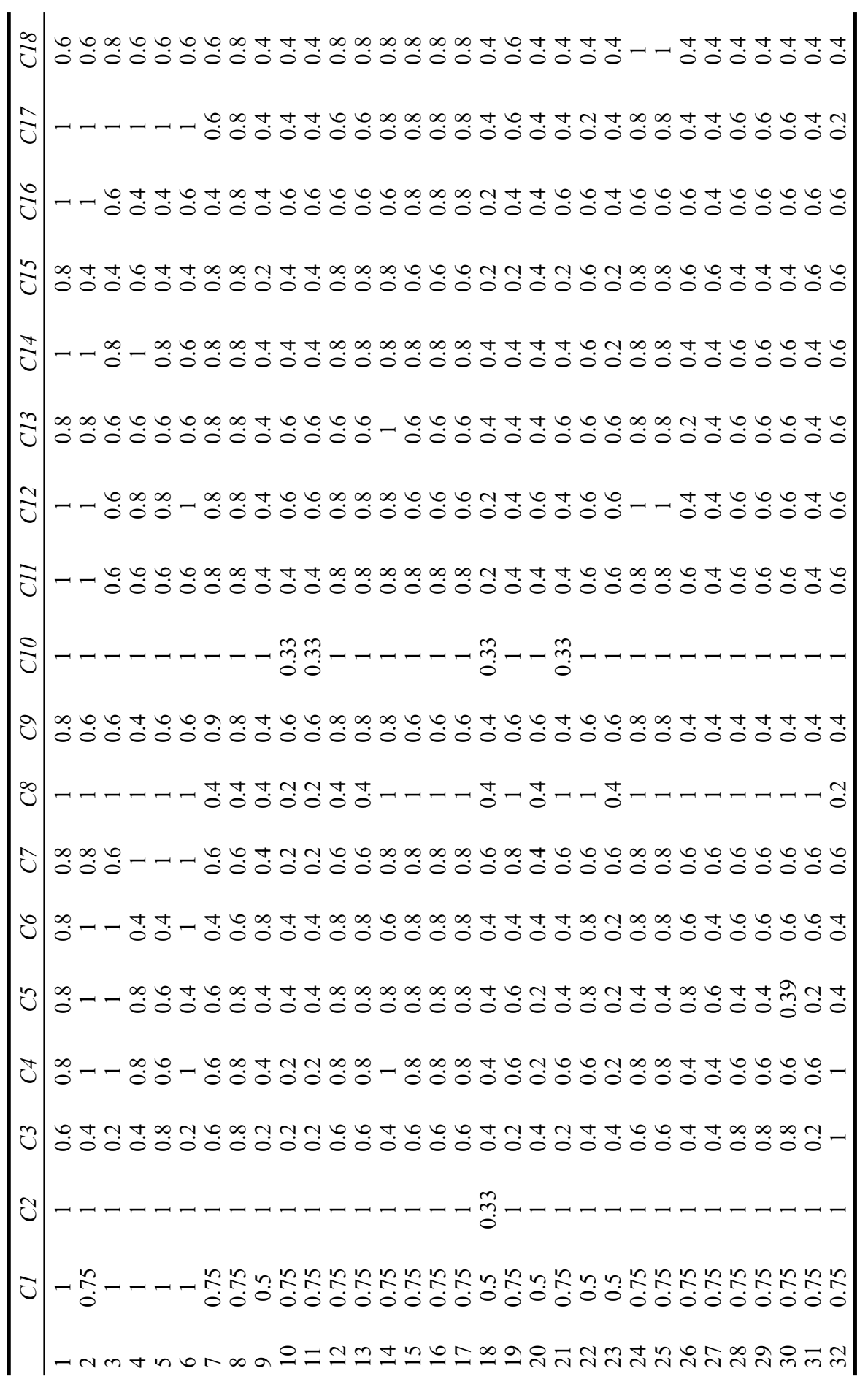


Table 4 Results for $\omega_{1}=\omega_{2}=0$

\begin{tabular}{|c|c|c|c|c|c|c|c|c|}
\hline \multirow{3}{*}{ Alternative } & \multicolumn{8}{|c|}{ MAOAM: net outstandingness, $p=q=\frac{1}{2} ; \omega_{1}=0 ; \omega_{2}=0$} \\
\hline & \multicolumn{2}{|c|}{ U-pair: $(P 1, N 1)$} & \multicolumn{2}{|c|}{ NB-pair: $(P 5, N 2)$} & \multicolumn{2}{|c|}{ PB-pair: $(P 4, N 1)$} & \multicolumn{2}{|c|}{ A-pair: $(P 3, N 3)$} \\
\hline & Value & Rank & Value & Rank & Value & Rank & Value & Rank \\
\hline 1 & 0.218 & 1 & 0.061 & 1 & 0.256 & 1 & 0.502 & 1 \\
\hline 2 & 0.214 & 2 & 0.02 & 2 & 0.233 & 2 & 0.472 & 2 \\
\hline 3 & 0.156 & 4 & -0.129 & 5 & 0.173 & 8 & 0.326 & 4 \\
\hline 4 & 0.085 & 14 & -0.296 & 14 & 0.117 & 13 & 0.155 & 14 \\
\hline 5 & 0.061 & 15 & -0.321 & 15 & 0.098 & 15 & 0.117 & 15 \\
\hline 6 & 0.087 & 13 & -0.265 & 13 & 0.106 & 14 & 0.178 & 13 \\
\hline 7 & 0.021 & 16 & -0.357 & 16 & 0.068 & 16 & 0.058 & 16 \\
\hline 8 & 0.135 & 5 & -0.117 & 4 & 0.183 & 4 & 0.313 & 5 \\
\hline 9 & -0.198 & 27 & -0.853 & 29 & -0.182 & 27 & -0.46 & 27 \\
\hline 10 & -0.215 & 28 & -0.847 & 27 & -0.199 & 28 & -0.471 & 28 \\
\hline 11 & -0.215 & 28 & -0.847 & 27 & -0.199 & 28 & -0.471 & 28 \\
\hline 12 & 0.101 & 11 & -0.24 & 11 & 0.149 & 11 & 0.204 & 11 \\
\hline 13 & 0.101 & 11 & -0.24 & 11 & 0.149 & 11 & 0.204 & 11 \\
\hline 14 & 0.165 & 3 & -0.11 & 3 & 0.202 & 3 & 0.345 & 3 \\
\hline 15 & 0.132 & 6 & -0.209 & 8 & 0.179 & 5 & 0.252 & 8 \\
\hline 16 & 0.132 & 6 & -0.209 & 8 & 0.179 & 5 & 0.252 & 8 \\
\hline 17 & 0.132 & 6 & -0.209 & 8 & 0.179 & 5 & 0.252 & 8 \\
\hline 18 & -0.28 & 32 & -0.891 & 32 & -0.262 & 32 & -0.562 & 32 \\
\hline 19 & -0.082 & 23 & -0.656 & 23 & -0.053 & 23 & -0.226 & 23 \\
\hline 20 & -0.232 & 30 & -0.879 & 31 & -0.215 & 30 & -0.508 & 31 \\
\hline 21 & -0.14 & 26 & -0.809 & 26 & -0.122 & 26 & -0.376 & 26 \\
\hline 22 & -0.011 & 17 & -0.531 & 18 & 0.027 & 17 & -0.083 & 18 \\
\hline 23 & -0.233 & 31 & -0.858 & 30 & -0.217 & 31 & -0.496 & 30 \\
\hline 24 & 0.124 & 9 & -0.197 & 6 & 0.163 & 9 & 0.252 & 6 \\
\hline 25 & 0.124 & 9 & -0.197 & 6 & 0.163 & 9 & 0.252 & 6 \\
\hline 26 & -0.059 & 22 & -0.623 & 22 & -0.027 & 22 & -0.186 & 22 \\
\hline 27 & -0.107 & 24 & -0.724 & 24 & -0.078 & 24 & -0.293 & 24 \\
\hline 28 & -0.022 & 19 & -0.582 & 19 & 0.013 & 18 & -0.125 & 19 \\
\hline 29 & -0.022 & 19 & -0.582 & 19 & 0.013 & 18 & -0.125 & 19 \\
\hline 30 & -0.023 & 21 & -0.588 & 21 & 0.011 & 20 & -0.13 & 21 \\
\hline 31 & -0.124 & 25 & -0.77 & 25 & -0.104 & 25 & -0.338 & 25 \\
\hline 32 & -0.021 & 18 & -0.511 & 17 & -0.003 & 21 & -0.081 & 17 \\
\hline
\end{tabular}

Furthermore, we observe that for all $\omega$ configurations, the various outstandingess pairs exhibit some variation in rankings despite that they often agree more than disagree overall.

In what concerns varying the $\omega$ values, we observe some variation in rankings for different outstandigness prdeicates pairs across the same $\omega$ configuration. But, across configurations, the rankings often agree more than disagree in this particular case. It seems that the effect of the Hernian means for large $\omega$ values is not as great as one expects. This led us to question the level of correlation among the attributes in our input data. We indeed measured the correlation between each pair of attributes and 
averaged them. The avarage mean correlation among attributes is indeed low being 0.564 . This findings partly explains the observed lack of significant variation among different rankings across various $\omega$ configurations.

Table 5 Results for $\omega_{1}=0, \omega_{2}=1,000$

\begin{tabular}{|c|c|c|c|c|c|c|c|c|}
\hline \multirow{3}{*}{ Alternative } & \multicolumn{8}{|c|}{ MAOAM: net outstandingness, $p=q=\frac{1}{2} ; \omega_{1}=0 ; \omega_{2}=1,000$} \\
\hline & \multicolumn{2}{|c|}{ U-pair: $(P 1, N 1)$} & \multicolumn{2}{|c|}{ NB-pair: $(P 5, N 2)$} & \multicolumn{2}{|c|}{ PB-pair: $(P 4, N 1)$} & \multicolumn{2}{|c|}{ A-pair: $(P 3, N 3)$} \\
\hline & Value & Rank & Value & Rank & Value & Rank & Value & Rank \\
\hline 1 & 0.216 & 1 & 0.095 & 1 & 0.251 & 1 & 0.5 & 1 \\
\hline 2 & 0.189 & 2 & 0.041 & 2 & 0.217 & 2 & 0.434 & 2 \\
\hline 3 & 0.119 & 10 & -0.185 & 7 & 0.144 & 10 & 0.253 & 7 \\
\hline 4 & 0.079 & 14 & -0.303 & 14 & 0.11 & 14 & 0.153 & 14 \\
\hline 5 & 0.07 & 15 & -0.31 & 15 & 0.107 & 15 & 0.14 & 15 \\
\hline 6 & 0.09 & 13 & -0.247 & 11 & 0.117 & 13 & 0.198 & 11 \\
\hline 7 & 0.039 & 16 & -0.33 & 16 & 0.081 & 16 & 0.072 & 16 \\
\hline 8 & 0.13 & 6 & -0.13 & 4 & 0.174 & 6 & 0.284 & 6 \\
\hline 9 & -0.177 & 27 & -0.88 & 30 & -0.167 & 27 & -0.419 & 27 \\
\hline 10 & -0.179 & 29 & -0.867 & 27 & -0.169 & 28 & -0.435 & 30 \\
\hline 11 & -0.179 & 29 & -0.867 & 27 & -0.169 & 28 & -0.435 & 30 \\
\hline 12 & 0.097 & 11 & -0.265 & 12 & 0.141 & 11 & 0.181 & 12 \\
\hline 13 & 0.097 & 11 & -0.265 & 12 & 0.141 & 11 & 0.181 & 12 \\
\hline 14 & 0.152 & 3 & -0.1 & 3 & 0.191 & 3 & 0.326 & 3 \\
\hline 15 & 0.12 & 7 & -0.228 & 8 & 0.163 & 7 & 0.24 & 8 \\
\hline 16 & 0.12 & 7 & -0.228 & 8 & 0.163 & 7 & 0.24 & 8 \\
\hline 17 & 0.12 & 7 & -0.228 & 8 & 0.163 & 7 & 0.24 & 8 \\
\hline 18 & -0.283 & 32 & -0.925 & 32 & -0.276 & 32 & -0.583 & 32 \\
\hline 19 & -0.071 & 23 & -0.7 & 23 & -0.051 & 23 & -0.199 & 23 \\
\hline 20 & -0.183 & 31 & -0.886 & 31 & -0.172 & 31 & -0.431 & 29 \\
\hline 21 & -0.128 & 26 & -0.848 & 26 & -0.116 & 26 & -0.351 & 26 \\
\hline 22 & -0.009 & 17 & -0.586 & 18 & 0.02 & 17 & -0.083 & 17 \\
\hline 23 & -0.178 & 28 & -0.871 & 29 & -0.17 & 30 & -0.419 & 28 \\
\hline 24 & 0.137 & 4 & -0.149 & 5 & 0.176 & 4 & 0.29 & 4 \\
\hline 25 & 0.137 & 4 & -0.149 & 5 & 0.176 & 4 & 0.29 & 4 \\
\hline 26 & -0.052 & 22 & -0.685 & 22 & -0.028 & 22 & -0.179 & 22 \\
\hline 27 & -0.093 & 24 & -0.775 & 24 & -0.072 & 24 & -0.265 & 24 \\
\hline 28 & -0.015 & 18 & -0.604 & 19 & 0.014 & 18 & -0.097 & 18 \\
\hline 29 & -0.015 & 18 & -0.604 & 19 & 0.014 & 18 & -0.097 & 18 \\
\hline 30 & -0.016 & 20 & -0.608 & 21 & 0.014 & 20 & -0.1 & 20 \\
\hline 31 & -0.097 & 25 & -0.801 & 25 & -0.084 & 25 & -0.28 & 25 \\
\hline 32 & -0.033 & 21 & -0.55 & 17 & -0.015 & 21 & -0.12 & 21 \\
\hline
\end{tabular}

\section{Concluding remarks}

In this paper, we introduced the MAOAM framework founded on two key concepts:

- Outstandingness predicates which are fuzzy predicates that measure the degree of outsandingness of an alternative against another alternative in both the positive 
and negative senses. We proposed different types of oustandingness pairs: U-pair, NB-pair, PB-pair, and A-pair and validated empirically their properties.

Table 6 Results for $\omega_{1}=1,000, \omega_{2}=0$

\begin{tabular}{|c|c|c|c|c|c|c|c|c|}
\hline \multirow{3}{*}{ Alternative } & \multicolumn{8}{|c|}{ MAOAM: net outstandingness, $p=q=\frac{1}{2} ; \omega_{1}=1,000 ; \omega_{2}=0$} \\
\hline & \multicolumn{2}{|c|}{ U-pair: $(P 1, N 1)$} & \multicolumn{2}{|c|}{ NB-pair: $(P 5, N 2)$} & \multicolumn{2}{|c|}{ PB-pair: $(P 4, N 1)$} & \multicolumn{2}{|c|}{ A-pair: $(P 3, N 3)$} \\
\hline & Value & Rank & Value & Rank & Value & Rank & Value & Rank \\
\hline 1 & 0.16 & 2 & 0.123 & 1 & 0.182 & 2 & 0.392 & 2 \\
\hline 2 & 0.18 & 1 & 0.063 & 2 & 0.187 & 1 & 0.406 & 1 \\
\hline 3 & 0.128 & 3 & -0.085 & 5 & 0.133 & 4 & 0.253 & 3 \\
\hline 4 & 0.059 & 14 & -0.239 & 14 & 0.072 & 14 & 0.075 & 14 \\
\hline 5 & 0.046 & 15 & -0.262 & 15 & 0.062 & 15 & 0.052 & 15 \\
\hline 6 & 0.075 & 11 & -0.216 & 13 & 0.08 & 13 & 0.116 & 11 \\
\hline 7 & 0.018 & 16 & -0.298 & 16 & 0.04 & 16 & 0.004 & 16 \\
\hline 8 & 0.094 & 5 & -0.05 & 4 & 0.123 & 5 & 0.21 & 5 \\
\hline 9 & -0.147 & 27 & -0.832 & 29 & -0.145 & 27 & -0.464 & 27 \\
\hline 10 & -0.165 & 28 & -0.819 & 27 & -0.162 & 28 & -0.479 & 28 \\
\hline 11 & -0.165 & 28 & -0.819 & 27 & -0.162 & 28 & -0.479 & 28 \\
\hline 12 & 0.064 & 12 & -0.169 & 11 & 0.089 & 11 & 0.107 & 12 \\
\hline 13 & 0.064 & 12 & -0.169 & 11 & 0.089 & 11 & 0.107 & 12 \\
\hline 14 & 0.118 & 4 & -0.047 & 3 & 0.137 & 3 & 0.247 & 4 \\
\hline 15 & 0.081 & 8 & -0.134 & 8 & 0.106 & 8 & 0.142 & 8 \\
\hline 16 & 0.081 & 8 & -0.134 & 8 & 0.106 & 8 & 0.142 & 8 \\
\hline 17 & 0.081 & 8 & -0.134 & 8 & 0.106 & 8 & 0.142 & 8 \\
\hline 18 & -0.223 & 32 & -0.871 & 32 & -0.221 & 32 & -0.551 & 32 \\
\hline 19 & -0.067 & 23 & -0.609 & 23 & -0.057 & 23 & -0.268 & 23 \\
\hline 20 & -0.182 & 30 & -0.859 & 31 & -0.179 & 30 & -0.507 & 31 \\
\hline 21 & -0.102 & 26 & -0.777 & 26 & -0.099 & 26 & -0.4 & 26 \\
\hline 22 & -0.022 & 21 & -0.474 & 17 & -0.008 & 21 & -0.142 & 18 \\
\hline 23 & -0.193 & 31 & -0.834 & 30 & -0.189 & 31 & -0.504 & 30 \\
\hline 24 & 0.087 & 6 & -0.133 & 6 & 0.107 & 6 & 0.158 & 6 \\
\hline 25 & 0.087 & 6 & -0.133 & 6 & 0.107 & 6 & 0.158 & 6 \\
\hline 26 & -0.055 & 22 & -0.574 & 22 & -0.044 & 22 & -0.229 & 22 \\
\hline 27 & -0.081 & 24 & -0.685 & 24 & -0.073 & 24 & -0.316 & 24 \\
\hline 28 & -0.013 & 18 & -0.529 & 19 & -0.001 & 18 & -0.168 & 19 \\
\hline 29 & -0.013 & 18 & -0.529 & 19 & -0.001 & 18 & -0.168 & 19 \\
\hline 30 & -0.016 & 20 & -0.536 & 21 & -0.005 & 20 & -0.173 & 21 \\
\hline 31 & -0.101 & 25 & -0.736 & 25 & -0.096 & 25 & -0.371 & 25 \\
\hline 32 & -0.005 & 17 & -0.474 & 18 & 0 & 17 & -0.113 & 17 \\
\hline
\end{tabular}

- Multi-variate (weighted) generalised Heronian means employed in the aggregation phase which are a family of multivariate generalised weighted Heronian means having as special cases the Beliakov et al.'s (2008) generalised Heronian means as well as that of Chu and Liu (2015). These types of means are especially useful when there is a significant correlation among attributes. We, however, through the proper parametrisation of these two means through $\omega_{1}$ and $\omega_{2}$ provide the 
flexibility to balance the proper level of compensation one should expect fromthese means. The application of these means on a real use case that lacks significant correlation among its attributes did not allow us to observe the results of such a diversity.

Table 7 Results for $\omega_{1}=1,000, \omega_{2}=1,000$

\begin{tabular}{|c|c|c|c|c|c|c|c|c|}
\hline \multirow{3}{*}{ Alternative } & \multicolumn{8}{|c|}{ MAOAM: net outstandingness, $p=q=\frac{1}{2} ; \omega_{1}=1,000 ; \omega_{2}=1,000$} \\
\hline & \multicolumn{2}{|c|}{ U-pair: $(P 1, N 1)$} & \multicolumn{2}{|c|}{ NB-pair: $(P 5, N 2)$} & \multicolumn{2}{|c|}{ PB-pair: $(P 4, N 1)$} & \multicolumn{2}{|c|}{ A-pair: $(P 3, N 3)$} \\
\hline & Value & Rank & Value & Rank & Value & Rank & Value & Rank \\
\hline 1 & 0.141 & 1 & 0.153 & 1 & 0.163 & 1 & 0.349 & 1 \\
\hline 2 & 0.123 & 2 & 0.084 & 2 & 0.136 & 2 & 0.296 & 2 \\
\hline 3 & 0.069 & 7 & -0.115 & 7 & 0.079 & 10 & 0.135 & 7 \\
\hline 4 & 0.042 & 14 & -0.214 & 14 & 0.053 & 14 & 0.058 & 14 \\
\hline 5 & 0.034 & 15 & -0.231 & 15 & 0.047 & 15 & 0.042 & 15 \\
\hline 6 & 0.05 & 13 & -0.17 & 11 & 0.059 & 13 & 0.089 & 11 \\
\hline 7 & 0.019 & 16 & -0.261 & 16 & 0.034 & 16 & 0.002 & 16 \\
\hline 8 & 0.079 & 4 & -0.042 & 4 & 0.102 & 4 & 0.17 & 4 \\
\hline 9 & -0.126 & 29 & -0.834 & 30 & -0.126 & 30 & -0.41 & 28 \\
\hline 10 & -0.116 & 27 & -0.809 & 27 & -0.115 & 27 & -0.419 & 30 \\
\hline 11 & -0.116 & 27 & -0.809 & 27 & -0.115 & 27 & -0.419 & 30 \\
\hline 12 & 0.052 & 11 & -0.172 & 12 & 0.071 & 11 & 0.078 & 12 \\
\hline 13 & 0.052 & 11 & -0.172 & 12 & 0.071 & 11 & 0.078 & 12 \\
\hline 14 & 0.087 & 3 & -0.036 & 3 & 0.107 & 3 & 0.189 & 3 \\
\hline 15 & 0.063 & 8 & -0.136 & 8 & 0.083 & 7 & 0.115 & 8 \\
\hline 16 & 0.063 & 8 & -0.136 & 8 & 0.083 & 7 & 0.115 & 8 \\
\hline 17 & 0.063 & 8 & -0.136 & 8 & 0.083 & 7 & 0.115 & 8 \\
\hline 18 & -0.211 & 32 & -0.886 & 32 & -0.21 & 32 & -0.56 & 32 \\
\hline 19 & -0.051 & 23 & -0.615 & 23 & -0.047 & 23 & -0.215 & 23 \\
\hline 20 & -0.13 & 31 & -0.84 & 31 & -0.129 & 31 & -0.417 & 29 \\
\hline 21 & -0.081 & 26 & -0.79 & 26 & -0.08 & 26 & -0.351 & 26 \\
\hline 22 & -0.01 & 17 & -0.486 & 17 & -0.001 & 17 & -0.119 & 17 \\
\hline 23 & -0.127 & 30 & -0.81 & 29 & -0.125 & 29 & -0.402 & 27 \\
\hline 24 & 0.078 & 5 & -0.069 & 5 & 0.096 & 5 & 0.163 & 5 \\
\hline 25 & 0.078 & 5 & -0.069 & 5 & 0.096 & 5 & 0.163 & 5 \\
\hline 26 & -0.037 & 22 & -0.599 & 22 & -0.032 & 22 & -0.198 & 22 \\
\hline 27 & -0.064 & 24 & -0.709 & 24 & -0.062 & 24 & -0.272 & 24 \\
\hline 28 & -0.013 & 18 & -0.523 & 19 & -0.006 & 18 & -0.137 & 18 \\
\hline 29 & -0.013 & 18 & -0.523 & 19 & -0.006 & 18 & -0.137 & 18 \\
\hline 30 & -0.014 & 20 & -0.527 & 21 & -0.007 & 20 & -0.14 & 20 \\
\hline 31 & -0.065 & 25 & -0.73 & 25 & -0.063 & 25 & -0.285 & 25 \\
\hline 32 & -0.024 & 21 & -0.492 & 18 & -0.019 & 21 & -0.158 & 21 \\
\hline
\end{tabular}

We have provided various pairs of outstandingness predicates alongside a taxonomy that could be further studied to align them with various decision-maker preferences in specifying how to measure outstandigness among alternatives. This might bring some added value with respect to existing method that focus more on outstandingness. Indeed, an possible future research direction is to deploy a carefully chosen outstandingness pairs for each attribute that describes more the fuzzy needs of the decision-maker on each attribute separately. 
In future works, more exhaustive experiments need to be done in order to fully understand the impact of the $\omega$ parameters especially when the attributes exhibit a high level of correlation. Finally, it remains an important and challenging task to find the proper combination of outstanding predicate pair as well as proper $\omega$ parameters for each specific application that would meet the decison-makers intentions and preferences.

\section{References}

Brans, J-P. and Mareschal, B. (2005) Promethee Methods, pp.163-186, Springer, New York, NY.

Bonferroni, C. (1950) 'Sulle medie multiple di potenze', Bollettino dell'Unione Matematica Italiana, December, Vol. 5, Nos. 3-4, pp.267-270.

Beliakov, G., Pradera, A. and Calvo, T. (2008) Aggregation Functions: A Guide for Practitioners, 1st ed., Springer Publishing Company Incorporated, Springer-Verlag, Berlin, Heidelberg.

Cacioppo, J.T. and Berntson, G.G. (1994) 'Interval-valued intuitionistic fuzzy heronian mean operators and their application in multi-criteria decision making', Psychological Bulletin, Vol. 115, No. 11, pp.401-423.

Cacioppo, J.T. and Berntson, G.G. (1999) 'The affect system: architecture and operating characteristics', Current Directions in Psychological Science, Vol. 8, No. 5, pp.133-137.

Cacioppo, J.T., Gardner, W.L. and Berntson, G.G. (1997) 'Beyond bipolar conceptualizations and measures: the case of attitudes and evaluative space', Personality and Social Psychology Review, Vol. 1, No. 1, pp.3-25, PMID: 15647126.

Czogała, E., Gottwald, S. and Pedrycz, W. (1982) 'Contribution to application of energy measure of fuzzy sets', Fuzzy Sets and Systems, Vol. 8, No. 2, pp.205-214.

Chu, Y. and Liu, P. (2015) 'Some two-dimensional uncertain linguistic heronian mean operators and their application in multiple-attribute decision making', Neural Computing and Applications, Vol. 26, No. 6, pp.1461-1480.

De Luca, A. and Termini, S. (1972) 'A definition of a nonprobabilistic entropy in the setting of fuzzy sets theory', Information and Control, Vol. 20, No. 4, pp.301-312.

Farr, R. and Brown, V.L. (1971) 'Evaluation and decision making', The Reading Teacher, Vol. 24, No. 4, pp.341-354.

Kaya, İ., Çolak, M. and Terzi, F. (2019) 'A comprehensive review of fuzzy multi criteria decision making methodologies for energy policy making', Energy Strategy Reviews, Vol. 24, pp.207-228 [online] https://doi.org/10.1016/j.esr.2019.03.003.

Janous, W. (2001) 'A note on generalized heronian means', Mathematical Inequalities \& Applications, Vol. 4, No. 3, pp.369-375.

Mao, X-B., Hu, S-S., Dong, J-Y., Wan, S-P. and Xu, G-L. (2018) 'Multi-attribute group decision making based on cloud aggregation operators under interval-valued hesitant fuzzy linguistic environment', International Journal of Fuzzy Systems, Vol. 20, No. 6, Article ID 145.

Norris, C., Gollan, J., Berntson, G. and Cacioppo, J. (2010) 'The current status of research on the structure of evaluative space', Biological Psychology, March, Vol. 84, pp.422-436.

Osgood, C.E., Suci, G.J. and Tenenbaum, P.H. (1957) The Measurement of Meaning, University of Illinois Press, Urbana.

Opricovic, S. and Tzeng, G-H. (2004) 'Compromise solution by mcdm methods: a comparative analysis of VIKOR and TOPSIS', European Journal of Operational Research, Vol. 156, No. 2, pp.445-455.

Opricovic, S. and Tzeng, G-H. (2007) 'Extended VIKOR method in comparison with outranking methods', European Journal of Operational Research, Vol. 178, No. 2, pp.514-529. 
Pramanik, S., Dey, P.P., Giri, B.C. and Smarandache, F. (2020) 'Bipolar neutrosophic projection based models for solving multi-attribute decision making problems', Neutrosophic Sets and Systems, September, Vol. 15, pp.70-79.

Papathanasiou, J. and Ploskas, N. (2018) Multiple Criteria Decision Aid: Methods, Examples and Python Implementations, Springer Optimization and Its Applications, Springer-Verlag, Berlin, Heidelberg.

Saaty, T.L. (1980) The Analytic Hierarchy Process: Planning, Priority Setting, Resource Allocation, McGraw-Hill International Book Co., New York, London.

San Cristóbal, J.R. (2011) 'Multi-criteria decision-making in the selection of a renewable energy project in Spain: the VIKOR method', Renewable Energy, Vol. 36, No. 2, pp.498-502.

Weber, S. (1984) 'Measures of fuzzy sets and measures of fuzziness', Fuzzy Sets and Systems, Vol. 13, No. 3, pp.247-271.

Wang, L., Zhang, H-Y. and Wang, J. (2017) 'Frank Choquet Bonferroni mean operators of bipolar neutrosophic sets and their application to multi-criteria decision-making problems', International Journal of Fuzzy Systems, August, Vol. 20, pp.1-16.

Xu, Y., Shang, X., Wang, J., Wu, W. and Huang, H. (2018) 'Some q-rung dual hesitant fuzzy heronian mean operators with their application to multiple attribute group decision-making', Symmetry, Vol. 10, No. 10, Article ID 472.

$\mathrm{Xu}, \mathrm{X}$. (2001) 'The sir method: a superiority and inferiority ranking method for multiple criteria decision making', European Journal of Operational Research, Vol. 131, No. 3, pp.587-602.

Xing, Y., Zhang, R., Wang, J. and Zhu, X. (2018) 'Some new pythagorean fuzzy choquet-frank aggregation operators for multi-attribute decision making', Int. J. Intell. Syst., Vol. 33, No. 11, pp.2189-2215.

Yazdani, M. and Graeml, F.R. (2014) 'VIKOR and its applications: a state-of-the-art survey', Int. J. Strateg. Decis. Sci., April, Vol. 5, No. 2, pp.56-83.

Ying, M. (1990) 'The alternativity measures of fuzzy sets', Fuzzy Sets and Systems, Vol. 37, No. 1, pp.105-110.

$\mathrm{Yu}$, D. and $\mathrm{Wu}, \mathrm{Y}$. (2012) 'Interval-valued intuitionistic fuzzy heronian mean operators and their application in multi-criteria decision making', Afr. J. Bus. Manage., Vol. 6, No. 11, pp.4158-4168.

Zhu, X., Bai, K., Wang, J., Zhang, R. and Xing, Y. (2019) 'Pythagorean fuzzy interaction power partitioned Bonferroni means with applications to multi-attribute group decision making', J. Intell. Fuzzy Syst., Vol. 36, No. 4, pp.3423-3438.

Zhang, R., Wang, J., Zhu, X., Xia, M. and Yu, M. (2017) 'Some generalized Pythagorean fuzzy Bonferroni mean aggregation operators with their application to multiattribute group decision-making', Complexity, Vol. 2017, Article ID 5937376, pp.1-16. 